\title{
Spinal Epidural Hematoma Related to Intracranial Hypotension
}

\author{
Kyung Han Cha, Tack Geun Cho, Chang Hyun Kim, Ho Kook Lee, Jae Gon Moon \\ Department of Neurosurgery, Hallym University Kangnam Sacred Heart Hospital, Seoul, Korea
}

\begin{abstract}
A 45-year-old female patient visited the hospital complaining of severe sudden headache and posterior neck pain. The patient did not have any traumatic history or abnormal neurologic finding. The patient had sudden quadriplegia and sensory loss. Cervical spine MRI scan was taken, and the compatible findings to acute epidural hematoma were shown. The emergency operation was performed. After the operation, the patient recovered all motor and senses. As there was CSF leakage in the postoperative wound, this was confirmed by cervical spinal computed tomography (CT). Then lumbar drainage was thus performed. The opening pressure upon lumbar puncture was not measured as it was very low. As a result of continous CSF leakage, dural repair was performed. After the operation, the patient had been discharged without neurologic deficits. In this case, it is sensible to suspect intracranial hypotension as a possible cause of spinal EDH.
\end{abstract}

Key Words: Spinal epidural hematoma $\cdot$ Headache $\cdot$ Neck pain $\cdot$ Cerebrospinal fluid leakage

\section{INTRODUCTION}

Spontaneous spinal epidural hematoma is a rare type of spinal epidural hematoma (SEDH) that occurs without specific cause. It typically manifests with sudden pain on the back or neck accompanied by sensory and motor paralysis symptoms, which progress fast according to the degree of the pressure on the spinal nerve ${ }^{1,218)}$. A person who shows these symptoms must be diagnosed immediately through spinal magnetic resonance imaging (MRI) and should undergo emergency surgery to obtain a good outcome $e^{5,9,11)}$.

SEDH is known to be related to coagulopathy, increased venous pressure, hypertension, vascular malformation, trauma, tumor, and an idiopathic origin ${ }^{2,12}$. No case showing a correlation between spinal epidural hematoma and a decrease in intracranial pressure, however, has yet been presented. Such a case will be presented in this report.

\section{CASE REPORT}

A 45-year-old female was admitted to the hospital after

- Received: June 15, 2013 • Revised: September 14, 2013

- Accepted: September 16, 2013

Corresponding Author: Tack Geun Cho, MD

Department of neurosurgery, Hallym University Kangnam Sacred Heart Hospital, Daerim-dong, Yeongdeungpo-gu, Seoul 150-950, Korea

Tel: +82-2-829-5175, Fax: +82-2-833-0219

E-mail: jotak01@naver.com complaining of an orthostatic headache and cervical pain the day before. The headache occurred whenever the patient was in a sitting or standing position, but not in a supine position. She did not have a history of trauma. She was alert and did not have other neurological symptoms. There were no abnormal findings in the brain MRI.

The day after the patient's hospital admission, she had sudden quadriplegia and paraesthesia. The motor grade of her lower extremities was 0 , and that of her upper extremities was I. The pain and high temperature on both sides were reduced, and the patient recovered with a motor grade of 3 in her upper and lower extremities. C-spine MRI was taken, and SEDH was suspected because isointensity was observed in the T1-weighted image of the epidural space from C2 to T2 while hyperintensity was observed in the T2-weighted image of the same (Fig. 1).

Angiography was performed to rule out any vascular abnormality, such as arteriovenous malformation. Its result was normal. An emergency left hemilaminectomy from C4 to T1 was performed to remove the hematoma, and controlled the bleeding. During the operation, engorged epidural veins were observed, and the dura was not damaged during the operation. After the operation, the patient recovered completely from motor and sensory deficits. 6 day after surgery, myelography and computed tomography (CT) were performed due to the CSF leakage in the wound. As a high density was observed from C3 to T3 (Fig. 2), lumbar drainage was performed. During the lumbar puncture, the opening pressure was too low to be measured, and the lumbar drainage was minimal. The patient was treated for two weeks, for stabilization. There 


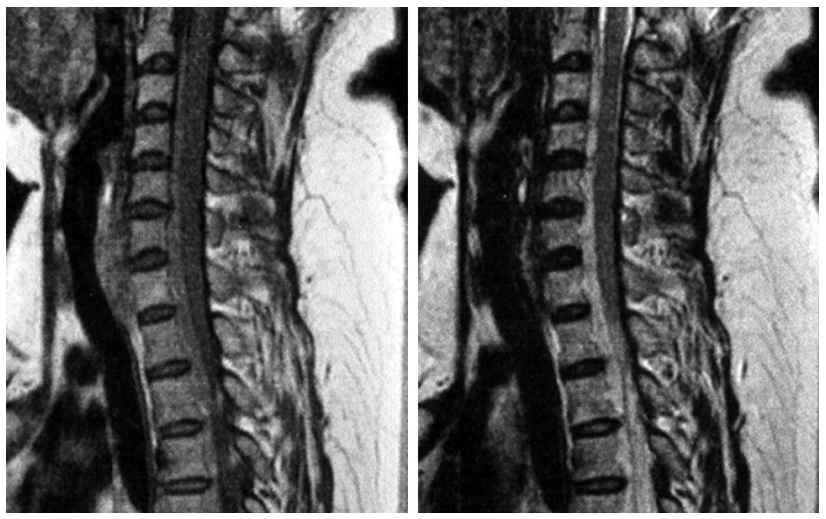

Fig. 1. Cenvical-spine MRI images: There is isointensity in the T1weighted image, and hyperintensity in the T2-weighted image, from the $\mathrm{C} 2$ to the $\mathrm{T} 2$ epidural space of the ventral side of the spinal cord.
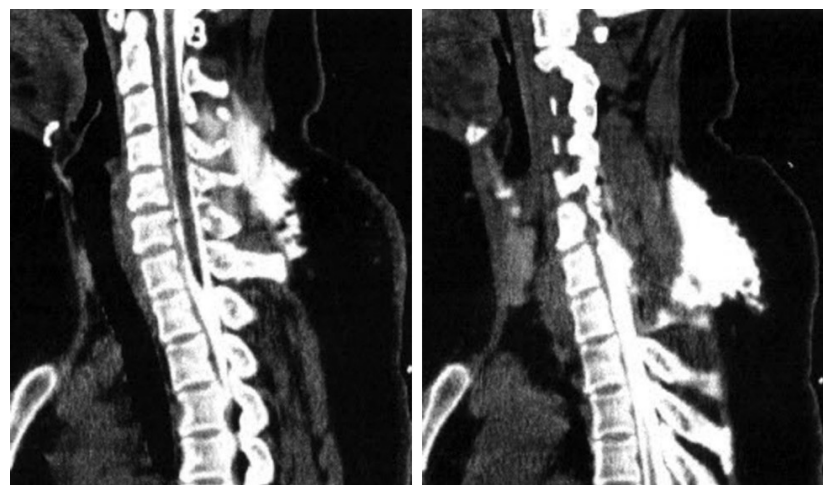

Fig. 2. Contrast-enhanced CT of cenvical-spine: There is fluid collection in the dorsal area of the vertebral bodies from C3 to T3 at 6 day after $1^{\text {st }}$ surgery.

was increased epidural fluid collection at C2 to T3 in the spinal CT (Fig. 3). Second operation was thus perform- ed, and there was no leakage at the dorsal part of the dura but there was some leakage at the C5-6 ventral part that was not found at the $1^{\text {st }}$ operation. The dura defect was covered with collagen sponge (Gelfoam) and fat tissue, and sealed with adhesive sealant (Tisseel). The symptoms disappeared, and no CSF leakage was found in the follow-up myelography and spinal CT. The patient was discharged without deficits.

\section{DISCUSSION}

Spontaneous SEDH is a very rare disease that occurs at a ratio of $0.1 / 100,000^{5)}$. It usually occurs in people in their $50 \mathrm{~s}$ to $80 \mathrm{~s}$, and more in males (male/female ratio, 1.4:1) ${ }^{4,10)}$. Although it can occur anywhere in the spinal cord, it mostly occurs in the dorsal spine, especially in the thoracolumbar and cervicothoracic region (the posterior and posterolateral
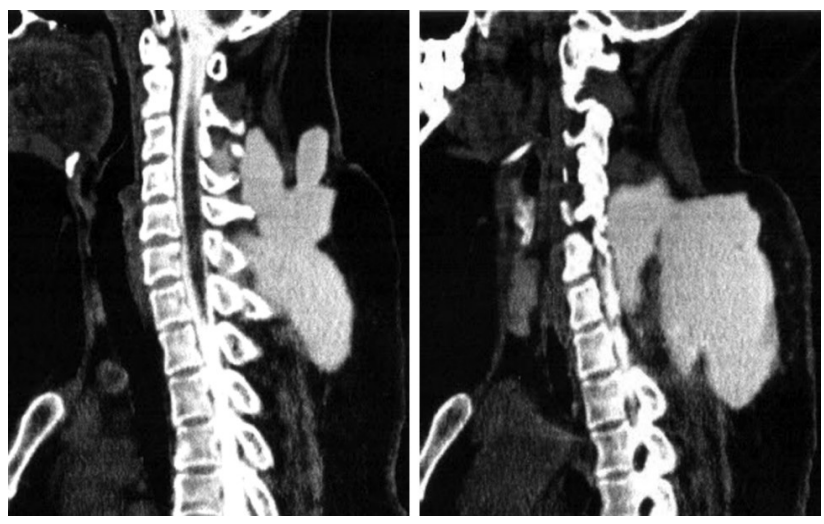

Fig. 3. Contrast-enhanced CT of cervical-spine: There is increased fluid collection in the dorsal area of the vertebral bodies from C2 to T3 at 24 day after $1^{\text {st }}$ surgery.

region of the spinal cord ${ }^{4,11,18)}$. It clinically manifests typically as sudden pain on the back or neck accompanied by sensory and motor paralysis symptoms, which progress fast according to the area and the degree of pressure on the spinal nerve $e^{28,9,11,18)}$. It can be diagnosed according to the patient's medical history and the results of the neurological examination, spinal cord angiography, CT, and MRI ${ }^{9,11)}$. MRI is the best choice, and the SEDH typically shows a heterogenous signal of a fusiform on the T2-weighted image, and isointensity on the T1- weighted image ${ }^{9,11)}$. Early operation is necessary as delayed operation can cause neurological defects and even death ${ }^{9,11)}$. There were the other cases that excellent functional recovery obtained after prompt surgical decompression ${ }^{7)}$. Although there have been recent attempts to conservatively treat patients with neurological symptom recovery or coagulopathy ${ }^{1,3)}$, the operation, when necessary, should not be delayed ${ }^{9,11)}$. The patient in this case had a sudden onset of orthostatic hypotension that progressed to sensory and motor paralysis. Although she recovered shortly, her recovery was incomplete. Also, she had an emergency operation as she had no abnormalities such as coagulopathy.

The clinical symptoms of intracranial hypotension include an orthostatic headache (its main symptom), pain or tetany on the column, nausea, vomiting, diplopia, tinnitus, and vertigo ${ }^{14,15}$. The known intracranial complications of intracranial hypotension include subdural hematoma or edema, and cerebellar tonsil herniation ${ }^{6,17}$. The intracranial hypotension also increases the risk of intracranial aneurysm ${ }^{16}$. Its diagnosis includes postural headache, diffuse pachymeningeal gadolinium enhancement, and low CSF opening pressure ${ }^{13)}$. There are various types of intracranial hypotension, however: one with a normal CSF pressure range, one without diffuse pachymeningeal enhancement, and one without orthostatic hypoten$\operatorname{sion}^{13,19)}$. Epidural fluid collection, engorgement of the epidu- 
ral vein, and abnormalities in the nerve root sleeve are observed in the spinal MRI ${ }^{13,14)}$. Conservative treatments include stabilization on a bed, analgesics, hydration, caffeine, mineralocorticoid, and glucocorticoid. Invasive treatments include autologous epidural blood patch and operation ${ }^{13,14,19}$.

In this case of postural headache, it is not sure whether it is due to CSF leakage, but there is a possibility of unnoticed surgical accident in the first surgery, causing in CSF leakage. However, there was no trace of damage to the dura mater, and engorgement of the intervertebral artery was seen. Thus, the reason may be due to a CSF leakage that was present even before the first surgery.

In the case, the patient complained of severe orthostatic headache for no reason, and cervical pain, at the first visit. Decreased intracranial pressure was suspected. In the first operation, engorgement and hyperemia of the spinal veins were found, but there was no CSF leakage. In the second operation, CSF leakage was directly observed. As the patient already had an earlier operation, epidural blood patch was not applied; instead, surgery was performed to block the CSF leakage. SEDH occurred sometime after the headache emerged. Also, decreased intracranial pressure was confirmed because the patient had orthostatic hypotension and lower CSF opening pressure when lumbar drainage was performed. Because the symotoms of patient and engorgement of spinal cord vein were associated with intrahypotension and there were no CSF leakage during the $1^{\text {st }}$ operation, the intracranial pressure decrease is thought to be the result of the slight CSF leakage, which may have led to the vascular congestion and hematoma in the spinal cord vein.

\section{CONCLUSION}

CSF leakage is a cause of intracranial hypotension, which can result in postural headache. It is difficult to state that an intracranial hypotension due to CSF leakage leads to spinal $\mathrm{EDH}$. However, in regards to this case, it is sensible to suspect intracranial hypotension as a possible cause of spinal EDH. When sensory and motor weaknesses are present along with postural headache, a brain lesion must be suspected. However, a lesion of the spinal cord must need attention.

\section{REFERENCES}

1. Azumagawa K, Yamamoto S, Tanaka K, Sakanaka H, Teraura $\mathrm{H}$, Takahashi K, et al: Non-operative treated spontaneous spinal epidural hematoma in a 12-year-old boy. Pediatr Emerg Care 28:167-169, 2012

2. Baek BS, Hur JW, Kwon KY, Lee HK: Spontaneous spinal epi- dural hematoma. J Korean Neurosurg Soc 44:40-42, 2008

3. Groen RJ: Non-operative treatment of spontaneous spinal epidural hematomas: a review of the literature and a comparison with operative cases. Acta Neurochir (Wien) 146:103-110, 2004

4. Groen RJ, van Alphen HA: Operative treatment of spontaneous spinal epidural hematomas: a study of the factors determining postoperative outcome. Neurosurgery 39:494-508; discussion 508-499, 1996

5. Holtas S, Heiling M, Lonntoft M: Spontaneous spinal epidural hematoma: findings at MR imaging and clinical correlation. Radiology 199:409-413, 1996

6. Kim BW, Jung YJ, Kim MS, Choi BY: Chronic subdural hematoma after spontaneous intracranial hypotension: a case treated with epidural blood patch on c1-2. J Korean Neurosurg Soc 50:274-276, 2011

7. Kim HW, Kwon YJ, Choi CS: Acute Spontaneous Spinal Epidural Hematoma. Kor J Spine 1:282-285, 2004

8. Kunizawa A, Fujioka M, Suzuki S, Ryu T, Asai A, Kawamoto $\mathrm{K}$, et al.: Spontaneous spinal epidural hematoma inducing acute anterior spinal cord syndrome. J Neurosurg Spine 10:574-577, 2009

9. Liao CC, Hsieh PC, Lin TK, Lin CL, Lo YL, Lee SC: Surgical treatment of spontaneous spinal epidural hematoma: a 5-year experience. J Neurosurg Spine 11:480-486, 2009

10. Liu Z, Jiao Q, Xu J, Wang X, Li S, You C: Spontaneous spinal epidural hematoma: analysis of 23 cases. Surg Neurol 69: 253260; discussion 260, 2008

11. Matsumura A, Namikawa T, Hashimoto R, Okamoto T, Yanagida I, Hoshi M, et al: Clinical management for spontaneous spinal epidural hematoma: diagnosis and treatment. Spine J 8:534-537, 2008

12. Miyagi Y, Miyazono M, Kamikaseda K: Spinal epidural vascular malformation presenting in association with a spontaneously resolved acute epidural hematoma. Case report. J Neurosurg 88: 909-911, 1998

13. Mokri B: Headaches caused by decreased intracranial pressure: diagnosis and management. Curr Opin Neurol 16:319-326, 2003

14. Park ES, Kim E: Spontaneous intracranial hypotension: clinical presentation, imaging features and treatment. J Korean Neurosurg Soc 45:1-4, 2009

15. Schievink WI, Maya MM: Frequency of intracranial aneurysms in patients with spontaneous intracranial hypotension. J Neurosurg 115:113-115, 2011

16. Schievink WI, Maya MM: Frequency of intracranial aneurysms in patients with spontaneous intracranial hypotension. Journal of Neurosurgery 115:113-115, 2011

17. Schievink WI, Maya MM, Nuno M: Chronic cerebellar hemorrhage in spontaneous intracranial hypotension: association with ventral spinal cerebrospinal fluid leaks: clinical article. J Neurosurg Spine 15:433-440, 2011

18. Son SN, Kang DH, Choi DS, Kim SK, Lim BH, Choi NC: A case of spontaneous spinal epidural hematoma mimicking a stroke. Neurologist 18:41-43, 2012

19. Spero M, Lazibat I, Stojic M, Vavro H: Normal pressure form of the spontaneous intracranial hypotension: a case report with pituitary enlargement and asymptomatic pituitary haemorrhage. Neurol Sci 32:933-935, 2011 